FACTA UNIVERSITATIS (NIŠ)

Ser. Math. Inform. Vol. 35, No 5 (2020), 1343-1356

https://doi.org/10.22190/FUMI2005343C

\title{
APPROXIMATION BY JAIN-SCHURER OPERATORS
}

\author{
Nursel Çetin and Gülen Başcanbaz-Tunca
}

(C) 2020 by University of Niš, Serbia | Creative Commons Licence: CC BY-NC-ND

Abstract. In this paper we deal with Jain-Schurer operators. We give an estimate, related to the degree of approximation, via moduli of smoothness of the first and the second order. Also, we present a Voronovskaja-type result. Moreover, we show that the Jain-Schurer operators preserve the properties of a modulus of continuity. Finally, we study monotonicity of the sequence of the Jain-Schurer operators when the attached function is convex and non-decreasing.

Keywords: Jain-Schurer operators; monotonicity; moduli of smoothness; Voronovskajatype result.

\section{Introduction}

In [19], Schurer constructed the following linear positive operators

$$
S_{n, p}(f ; x)=e^{-(n+p) x} \sum_{k=0}^{\infty} f\left(\frac{k}{n}\right) \frac{(n+p)^{k} x^{k}}{k !},
$$

where $x \in[0, b], b<\infty, n \in \mathbb{N}, p \geq 0$, and $f$ is real valued and bounded function on $[0, \infty)$. The case $p=0$ gives the the well known Szász-Mirakjan operators. There are a number of generalizations of Szász-Mirakjan operators, here we cite only a few ([4], [6], [10], [11]) with references therein. Some works concerning Schurer's setting can be found in [3], [14], [20], [16] and [17]. Motivated by these statements, we extend the well known Jain operators in the Schurer's design. Recall that in [12], Jain constructed the following linear positive operators

$$
P_{n}^{[\beta]}(f ; x)=\sum_{k=0}^{\infty} f\left(\frac{k}{n}\right) w_{\beta}(k ; n x), \quad x \in(0, \infty),
$$

Received March 12, 2019; accepted February 11, 2020

2020 Mathematics Subject Classification. Primary 41A25; Secondary 41A36 
and $P_{n}^{[\beta]}(f ; 0)=f(0)$, where $n \in \mathbb{N}, \beta \in[0,1), f \in C[0, \infty)$, and for $0<\alpha<$ $\infty, w_{\beta}(k ; \alpha)$ is given by

$$
w_{\beta}(k ; \alpha):=\frac{\alpha(\alpha+k \beta)^{k-1}}{k !} e^{-(\alpha+k \beta)}, \quad k \in \mathbb{N} \cup\{0\}
$$

and it satisfies $\sum_{k=0}^{\infty} w_{\beta}(k ; \alpha)=1$. In the paper, the author studied convergence properties and the order of approximation by the sequence of these operators on any finite closed interval of $[0, \infty)$ by taking $\beta$ as a sequence $\beta_{n}$ such that $\beta_{n} \rightarrow 0$ as $n \rightarrow \infty$. For some interesting papers concerning Jain operators, we refer to [1], [2], [7], [9], [18], [23] and references therein. Obviously, the case $\beta=0$ gives the well known Szász-Mirakjan operators [22].

In this work, for a fixed $p \in \mathbb{N} \cup\{0\}$, we consider the linear positive operators denoted by $S_{n, p}^{\beta}, n \in \mathbb{N}$, and defined as

$$
S_{n, p}^{\beta}(f ; x)=\sum_{k=0}^{\infty} f\left(\frac{k}{n}\right) w_{\beta}(k ;(n+p) x), \quad x \in(0, \infty)
$$

and $S_{n, p}^{\beta}(f ; 0)=f(0)$, for $f \in C_{B}[0, \infty):=\{f \in C[0, \infty): f$ is bounded $\}$, $\beta \in[0,1)$, and $w_{\beta}(k ;(n+p) x)$ given by (1.3). We call $S_{n, p}^{\beta}$ as Jain-Schurer operators. Note that, each $S_{n, p}^{\beta}$ maps $C_{B}[0, \infty)$ into itself, and the case $p=0$ covers the Jain operators: $S_{n, 0}^{\beta}=P_{n}^{[\beta]}, n \in \mathbb{N}$. On the other hand, in the case $\beta=0, S_{n, p}^{\beta}$ reduces to the Schurer extension of the Szász-Mirakjan operators given by (1.1). We obtain an estimate, which will be used next for the rate of convergence, with the help of the modulus of smoothness of a bounded and continuous function, and prove a Voronovskaja-type result. Moreover, we show that each JainSchurer operator preserves the properties of a general modulus of continuity. Finally, we investigate the monotonicity of the sequence of the Jain-Schurer operators $S_{n, p}^{\beta}(f)$, with respect to $n$, when the function $f$ is convex and non-decreasing.

Now, denoting $e_{j}(t)=t^{j}, j \in \mathbb{N} \cup\{0\}$ and $\varphi_{x}^{j}(t):=(t-x)^{j}, j \in \mathbb{N}$, for the Jain operators $P_{n}^{[\beta]}$ we have (see, e.g., [11, Lemma 1])

Lemma 1.1. For the operators $P_{n}^{[\beta]}$ given by (1.2), one has

$$
\begin{aligned}
& P_{n}^{[\beta]}\left(e_{0} ; x\right)=1 \\
& P_{n}^{[\beta]}\left(e_{1} ; x\right)=\frac{x}{1-\beta}, \\
& P_{n}^{[\beta]}\left(e_{2} ; x\right)=\frac{x^{2}}{(1-\beta)^{2}}+\frac{x}{n(1-\beta)^{3}}, \\
& P_{n}^{[\beta]}\left(e_{3} ; x\right)=\frac{x^{3}}{(1-\beta)^{3}}+\frac{3 x^{2}}{n(1-\beta)^{4}}+\frac{(1+2 \beta) x}{n^{2}(1-\beta)^{5}}, \\
& P_{n}^{[\beta]}\left(e_{4} ; x\right)=\frac{x^{4}}{(1-\beta)^{4}}+\frac{6 x^{3}}{n(1-\beta)^{5}}+\frac{(8 \beta+7) x^{2}}{n^{2}(1-\beta)^{6}}+\frac{\left(6 \beta^{2}+8 \beta+1\right) x}{n^{3}(1-\beta)^{7}} .
\end{aligned}
$$


Making use of Lemma 1.1, straightforward computation shows that moments and central moments of the Jain-Schurer operators are obtained as in the following lemmas, respectively:

Lemma 1.2. For the operators $S_{n, p}^{\beta}$ given by (1.4), one has

$$
S_{n, p}^{\beta}\left(e_{j} ; x\right)=P_{n}^{[\beta]}\left(e_{j} ;\left(\frac{n+p}{n}\right) x\right), \quad j=0,1, \ldots
$$

Lemma 1.3. For the operators $S_{n, p}^{\beta}$ given by (1.4), one has

$$
\begin{aligned}
S_{n, p}^{\beta}\left(\varphi_{x}^{1} ; x\right)= & \left(\beta+\frac{p}{n}\right) \frac{x}{1-\beta}, \\
S_{n, p}^{\beta}\left(\varphi_{x}^{2} ; x\right)= & \left(\beta+\frac{p}{n}\right)^{2} \frac{x^{2}}{(1-\beta)^{2}}+\left(1+\frac{p}{n}\right) \frac{x}{n(1-\beta)^{3}}, \\
S_{n, p}^{\beta}\left(\varphi_{x}^{4} ; x\right)= & \left(\beta+\frac{p}{n}\right)^{4} \frac{x^{4}}{(1-\beta)^{4}}+6\left(\beta+\frac{p}{n}\right)^{2}\left(1+\frac{p}{n}\right) \frac{x^{3}}{n(1-\beta)^{5}} \\
& +\left(1+\frac{p}{n}\right) \frac{\left(4 n \beta+3 n+8 p \beta+7 p+8 n \beta^{2}\right)}{n^{3}(1-\beta)^{6}} x^{2}+\left(1+\frac{p}{n}\right) \frac{\left(6 \beta^{2}+8 \beta+1\right)}{n^{3}(1-\beta)^{7}} x .
\end{aligned}
$$

\section{Modulus of smoothness $K$-Functional}

In this part of the paper, we extend the result proved by Agratini for the Jain operators [2, Theorem 2] to the Jain-Schurer operators. To this aim, we recall the terminology that will be used in the results. As usual, let $C_{B}[0, \infty)$ denote the space of real valued, bounded and continuous functions defined on $[0, \infty)$ equipped with the norm given by

$$
\|f\|=\sup _{x \in[0, \infty)}|f(x)|
$$

for $f \in C_{B}[0, \infty)$. Also, let $U C_{B}[0, \infty)$ denote the space of all real valued bounded and uniformly continuous functions on $[0, \infty)$.

For a bounded, real valued function $f$ on $[0, \infty)$ and $\delta>0$, the first modulus of smoothness, modulus of continuity, of $f$ is defined by

$$
\omega_{1}(f, \delta)=\sup _{|h| \leq \delta x, x+h \in[0, \infty)} \sup _{|f(x+h)-f(x)|} \mid f(x)
$$

and second modulus of smoothness of $f$ is defined by

$$
\omega_{2}(f, \delta)=\sup _{|h| \leq \delta} \sup _{x+2 h \in[0, \infty)}|f(x+2 h)-2 f(x+h)+f(x)| .
$$

We have the following well known property of the modulus of smoothness (see, e.g., [3, p. 266, Lemma 5.1.1]). 
Remark 2.1. If $f \in U C_{B}[0, \infty)$, then $\lim _{\delta \rightarrow 0^{+}} \omega_{k}(f, \delta)=0$ for $k=1,2$.

For convenience, we need the following Peetre's $K$-functional defined by

$$
K(f, \delta)=\inf _{g \in C_{B}^{2}[0, \infty)}\left\{\|f-g\|+\delta\left\|g^{\prime \prime}\right\|\right\}
$$

where $\delta>0$ and

$$
C_{B}^{2}[0, \infty)=\left\{g \in C_{B}[0, \infty): g^{\prime}, g^{\prime \prime} \in C_{B}[0, \infty)\right\} .
$$

Note that the modulus of smoothness and the $K$-functional of an $f \in C_{B}[0, \infty)$ are related to each other as in the following sense: There exist positive constants $C_{1}$ and $C_{2}$ such that

$$
C_{1} \omega_{2}(f, \delta) \leq K\left(f, \delta^{2}\right) \leq C_{2} \omega_{2}(f, \delta)
$$

(see, e.g., [8, p. 177, Theorem 2.4]).

Below, we present a quantitative estimate to reach to the subsequent result concerning the rate of the approximation by $\left\{S_{n, p}^{\beta_{n}}(f ; x)\right\}_{n \geq 1}$.

Theorem 2.1. Let $p \in \mathbb{N}_{0}$ be fixed, $0 \leq \beta<1$ and $f \in C_{B}[0, \infty)$. Then, for each $x \in(0, \infty)$, one has

$$
\left|S_{n, p}^{\beta}(f ; x)-f(x)\right| \leq \omega_{1}\left(f,\left(\beta+\frac{p}{n}\right) \frac{x}{1-\beta}\right)+C \omega_{2}\left(f, \delta_{n, p}^{\beta}(x)\right),
$$

where $C>0$ is a positive constant and

$$
\delta_{n, p}^{\beta}(x):=\frac{1}{2} \sqrt{\left(\beta+\frac{p}{n}\right)^{2} \frac{x^{2}}{(1-\beta)^{2}}+\left(1+\frac{p}{n}\right) \frac{x}{2 n(1-\beta)^{3}}} .
$$

Proof. Consider an auxiliary operator

$$
\bar{S}_{n, p}^{\beta}(f ; x):=S_{n, p}^{\beta}(f ; x)+f(x)-f\left(\left(1+\frac{p}{n}\right) \frac{x}{1-\beta}\right)
$$

for $f \in C_{B}[0, \infty), n \in \mathbb{N}$. In this case, $\bar{S}_{n, p}^{\beta}$ are linear and positive and each operator preserves the linear functions. Now, let $g \in C_{B}^{2}[0, \infty)$. From Taylor's formula about an arbitrary fixed point $x$, one has

$$
g(t)=g(x)+g^{\prime}(x)(t-x)+\int_{x}^{t}(t-y) g^{\prime \prime}(y) d y
$$

for $t \in[0, \infty)$. Application of the operators $\bar{S}_{n, p}^{\beta}$ on both sides of (2.5) gives that

$$
\bar{S}_{n, p}^{\beta}(g ; x)-g(x)=g^{\prime}(x) \bar{S}_{n, p}^{\beta}(t-x ; x)+\bar{S}_{n, p}^{\beta}\left(\int_{x}^{t}(t-y) g^{\prime \prime}(y) d y ; x\right) .
$$


Taking (2.4) into account for $f(t)=\int_{x}^{t}(t-y) g^{\prime \prime}(y) d y$, expression (2.6) reduces to

$\bar{S}_{n, p}^{\beta}(g ; x)-g(x)=S_{n, p}^{\beta}\left(\int_{x}^{t}(t-y) g^{\prime \prime}(y) d y ; x\right)-\int_{x}^{\left(1+\frac{p}{n}\right) \frac{x}{1-\beta}}\left[\left(1+\frac{p}{n}\right) \frac{x}{1-\beta}-y\right] g^{\prime \prime}(y) d y$.

Using the fact

$$
\left|\int_{x}^{\left(1+\frac{p}{n}\right) \frac{x}{1-\beta}}\left[\left(1+\frac{p}{n}\right) \frac{x}{1-\beta}-y\right] g^{\prime \prime}(y) d y\right| \leq \frac{1}{2}\left(S_{n, p}^{\beta}\left(\varphi_{x}^{1} ; x\right)\right)^{2}\left\|g^{\prime \prime}\right\|,
$$

by Lemma 1.3 , we obtain

$$
\begin{aligned}
& \left|\bar{S}_{n, p}^{\beta}(g ; x)-g(x)\right| \\
\leq & S_{n, p}^{\beta}\left(\left|\int_{x}^{t}(t-y) g^{\prime \prime}(y) d y\right| ; x\right)+\left|\int_{x}^{\left(1+\frac{p}{n}\right) \frac{x}{1-\beta}}\left[\left(1+\frac{p}{n}\right) \frac{x}{1-\beta}-y\right]\right| g^{\prime \prime}(y)|d y| \\
\leq & \frac{\left\|g^{\prime \prime}\right\|}{2}\left[S_{n, p}^{\beta}\left(\varphi_{x}^{2} ; x\right)+\left(S_{n, p}^{\beta}\left(\varphi_{x}^{1} ; x\right)\right)^{2}\right] \\
(2.7) & \frac{\left\|g^{\prime \prime}\right\|}{2}\left[2\left(\beta+\frac{p}{n}\right)^{2} \frac{x^{2}}{(1-\beta)^{2}}+\left(1+\frac{p}{n}\right) \frac{x}{n(1-\beta)^{3}}\right] .
\end{aligned}
$$

On the other hand, from (2.4) and Lemma 1.2, it can be easily obtained that

$$
\left|\bar{S}_{n, p}^{\beta}(f ; x)\right| \leq\left|S_{n, p}^{\beta}(f ; x)\right|+2\|f\| \leq 3\|f\|
$$

for $f \in C_{B}[0, \infty)$. Thus, taking (2.4), (2.7) and (2.8) into account, for $f, g \in$ $C_{B}[0, \infty)$ one has

$$
\begin{aligned}
& \left|S_{n, p}^{\beta}(f ; x)-f(x)\right| \\
\leq & \left|\bar{S}_{n, p}^{\beta}(f-g ; x)-(f-g)(x)\right|+\left|\bar{S}_{n, p}^{\beta}(g ; x)-g(x)\right| \\
& +\left|f(x)-f\left(\left(1+\frac{p}{n}\right) \frac{x}{1-\beta}\right)\right| \\
\leq & \omega_{1}\left(f,\left(\beta+\frac{p}{n}\right) \frac{x}{1-\beta}\right) \\
& +4\left\{\|f-g\|+\frac{1}{4}\left[\left(\beta+\frac{p}{n}\right)^{2} \frac{x^{2}}{(1-\beta)^{2}}+\left(1+\frac{p}{n}\right) \frac{x}{2 n(1-\beta)^{3}}\right]\left\|g^{\prime \prime}\right\|\right\} .
\end{aligned}
$$


Finally, taking infimum over all $g \in C_{B}^{2}[0, \infty)$ on the right hand-side of the last inequality and applying (2.1), we get

$$
\begin{aligned}
\left|S_{n, p}^{\beta}(f ; x)-f(x)\right| & \leq \omega_{1}\left(f,\left(\beta+\frac{p}{n}\right) \frac{x}{1-\beta}\right)+K\left(f,\left(\delta_{n, p}^{\beta}(x)\right)^{2}\right) \\
& \leq \omega_{1}\left(f,\left(\beta+\frac{p}{n}\right) \frac{x}{1-\beta}\right)+C \omega_{2}\left(f, \delta_{n, p}^{\beta}(x)\right),
\end{aligned}
$$

where $\delta_{n, p}^{\beta}(x)$ is given by $(2.3)$.

Note that the case $p=0$ in the above theorem reduces to Theorem 2 in [2].

Taking Remark 2.1 and (2.2) into account, we reach to the following conclusion:

Corollary 2.1. i) If $\beta$ is taken as a sequence $\beta_{n}$ such that $0 \leq \beta_{n}<1$ and $\lim _{n \rightarrow \infty} \beta_{n}=0$ and $f \in U C_{B}[0, \infty)$, then one gets $\lim _{n \rightarrow \infty} S_{n, p}^{\beta_{n}}(f ; x)=f(x)$ on $[0, \infty)$ and the order of the approximation does not exceed to that of $\omega_{1}\left(f,\left(\beta_{n}+\frac{p}{n}\right) \frac{x}{1-\beta_{n}}\right)+$ $C \omega_{2}\left(f, \delta_{n, p}^{\beta_{n}}(x)\right)$.

ii) If $\beta$ is taken as a sequence $\beta_{n}$ such that $0 \leq \beta_{n}<1$ and $\lim _{n \rightarrow \infty} \beta_{n}=0$ and $f \in C_{B}[0, \infty)$, then $\left\{S_{n, p}^{\beta_{n}}(f)\right\}_{n \geq 1}$ converges uniformly to $f$ on $[a, b], 0 \leq a<b<$ $\infty$, by the well known Korovkin theorem.

\section{A Voronovskaja-type result}

In [9], Farcaş obtained the following Voronovskaja-type result for the Jain operator $P_{n}^{[\beta]}$ given by $(1.2)$ :

$$
\lim _{n \rightarrow \infty} n\left\{P_{n}^{\left[\beta_{n}\right]}(f ; x)-f(x)\right\}=\frac{x}{2} f^{\prime \prime}(x), \quad x>0,
$$

for $f \in C_{2}[0, \infty)$, the space of all continuous functions having continuous second order derivative, where $0 \leq \beta_{n}<1$ is a sequence such that $\lim _{n \rightarrow \infty} \beta_{n}=0$.

Note that a Voronovskaja-type result for a generalization of the Jain operators was obtained by Olgun et al. [18]. On the other hand, a Voronovskaja-type theorem as well as its a generalized form for Schurer setting of the Szász-Mirakjan operators were obtained by Sikkema in [20, p. 333].

In this part, we investigate a Voronovskaja-type result for the Jain-Schurer operators $S_{n, p}^{\beta}, n \in \mathbb{N}$.

Theorem 3.1. Let $p \in \mathbb{N}_{0}$ be fixed and $0 \leq \beta_{n}<1$ be a sequence such that $\lim _{n \rightarrow \infty} n \beta_{n}=0$. If $f$ is bounded and continuous on $[0, \infty)$ and has the second order derivative at some $x \in(0, \infty)$, then one has

$$
\lim _{n \rightarrow \infty} n\left\{S_{n, p}^{\beta_{n}}(f ; x)-f(x)\right\}=p x f^{\prime}(x)+\frac{x}{2} f^{\prime \prime}(x) .
$$


Proof. From Taylor's formula, one has

$$
f(t)=f(x)+f^{\prime}(x)(t-x)+\frac{1}{2} f^{\prime \prime}(x)(t-x)^{2}+h(t-x)(t-x)^{2},
$$

at the fixed point $x \in[0, \infty)$, where $h(t-x)$ is bounded for all $t \in[0, \infty)$ and $\lim _{t \rightarrow x} h(t-x)=0$. Application of the operators $S_{n, p}^{\beta}$ to (3.1) implies

$$
\begin{aligned}
n\left[S_{n, p}^{\beta_{n}}(f ; x)-f(x)\right]= & f^{\prime}(x) n S_{n, p}^{\beta_{n}}(t-x ; x)+\frac{1}{2} f^{\prime \prime}(x) n S_{n, p}^{\beta_{n}}\left((t-x)^{2} ; x\right) \\
& +n S_{n, p}^{\beta_{n}}\left(h(t-x)(t-x)^{2} ; x\right) .
\end{aligned}
$$

Using the facts $\lim _{n \rightarrow \infty} n \beta_{n}=0$ and Lemma 1.3, it readily follows that

$$
\lim _{n \rightarrow \infty} n S_{n, p}^{\beta_{n}}(t-x ; x)=p x
$$

and

$$
\lim _{n \rightarrow \infty} n S_{n, p}^{\beta_{n}}\left((t-x)^{2} ; x\right)=x .
$$

Hence, we have

$\lim _{n \rightarrow \infty} n\left(S_{n, p}^{\beta_{n}}(f ; x)-f(x)\right)=p x f^{\prime}(x)+\frac{x}{2} f^{\prime \prime}(x)+\lim _{n \rightarrow \infty} n S_{n, p}^{\beta_{n}}\left(h(t-x)(t-x)^{2} ; x\right)$.

It suffices to prove that $\lim _{n \rightarrow \infty} n S_{n, p}^{\beta_{n}}\left(h(t-x)(t-x)^{2} ; x\right)=0$. Indeed, defining $h(0)=0$ and taking the fact $\lim _{t \rightarrow x} h(t-x)=0$ into account, we get that $h$ is continuous at $x$. Hence, for each $\varepsilon>0$, there is a $\delta>0$ such that $|h(t-x)|<$ $\varepsilon$ for all $t$ satisfying $|t-x|<\delta$. On the other hand, since $h(t-x)$ is bounded on $[0, \infty)$, there is an $M>0$ such that $|h(t-x)| \leq M$ for all $t$. Therefore, we may write $|h(t-x)| \leq M \frac{(t-x)^{2}}{\delta^{2}}$ when $|t-x| \geq \delta$. So, these arguments enable one to write $|h(t-x)| \leq \varepsilon+M \frac{(t-x)^{2}}{\delta^{2}}$ for all $t$. The monotonicity and linearity of $S_{n, p}^{\beta_{n}}$ give that

$$
\begin{aligned}
S_{n, p}^{\beta_{n}}\left(h(t-x)(t-x)^{2} ; x\right) & \leq \varepsilon S_{n, p}^{\beta_{n}}\left((t-x)^{2} ; x\right)+\frac{M}{\delta^{2}} S_{n, p}^{\beta_{n}}\left((t-x)^{4} ; x\right) \\
& =\varepsilon S_{n, p}^{\beta_{n}}\left(\varphi_{x}^{2} ; x\right)+\frac{M}{\delta^{2}} S_{n, p}^{\beta_{n}}\left(\varphi_{x}^{4} ; x\right) .
\end{aligned}
$$

Making use of Lemma 1.3, with $\beta=\beta_{n}$,

$$
\lim _{n \rightarrow \infty} n S_{n, p}^{\beta_{n}}\left(h(t-x)(t-x)^{2} ; x\right)=0
$$

by the hypothesis on $\beta_{n}$, which completes the proof. 


\section{A Retaining Property}

Recall that $A$ continuous and non-negative function $\omega$ defined on $[0, \infty)$ is called a modulus of continuity, if each of the following conditions is satisfied:

i) $\omega(u+v) \leq \omega(u)+\omega(v)$ for $u, v, u+v \in[0, \infty)$, i.e., $\omega$ is semi-additive,

ii) $\omega(u) \geq \omega(v)$ for $u \geq v>0$, i.e., $\omega$ is non-decreasing,

iii) $\lim _{u \rightarrow 0^{+}} \omega(u)=\omega(0)=0$, ([15, p. 106]).

In [13], Li proved that each Bernstein polynomial preserves the properties of modulus of continuity on $[0,1]$. Motivated by this result, in this section we will show that each Jain-Schurer operator has this preservation property as well. In the proof, we need the following Jensen formula

$$
(u+v)(u+v+m \beta)^{m-1}=\sum_{k=0}^{m}\left(\begin{array}{c}
m \\
k
\end{array}\right) u(u+k \beta)^{k-1} v[v+(m-k) \beta]^{m-k-1}
$$

where $u, v$, and $\beta \in \mathbb{R}$ (see, e.g., [3, p. 326]).

Theorem 4.1. Let $p \in \mathbb{N}_{0}$ be fixed and $0 \leq \beta<1$. If $\omega$ is a bounded modulus of continuity on $[0, \infty)$, then for each $n \in \mathbb{N}, S_{n, p}^{\beta}(\omega ; x)$ is also a modulus of continuity.

Proof. Let $x, y \in[0, \infty)$ and $x \leq y$. From the definition of $S_{n}^{\beta}$, we have

$$
S_{n, p}^{\beta}(\omega ; y)=\sum_{j=0}^{\infty} \omega\left(\frac{j}{n}\right) \frac{(n+p) y[(n+p) y+j \beta]^{j-1}}{j !} e^{-[(n+p) y+j \beta]} .
$$

Taking $u=(n+p) x, v=(n+p) y-(n+p) x$ and $m=j$ in (4.1), we obtain

$$
\begin{aligned}
& (n+p) y((n+p) y+j \beta)^{j-1} \\
= & \sum_{k=0}^{j}\left(\begin{array}{l}
j \\
k
\end{array}\right)(n+p) x[(n+p) x+k \beta]^{k-1} \\
& \times(n+p)(y-x)[(n+p)(y-x)+(j-k) \beta]^{j-k-1} .
\end{aligned}
$$


Substituting this expression into (4.2) we get

$$
\begin{aligned}
S_{n, p}^{\beta}(\omega ; y)= & \sum_{j=0}^{\infty} \sum_{k=0}^{j} \omega\left(\frac{j}{n}\right)\left(\begin{array}{l}
j \\
k
\end{array}\right) \frac{1}{j !}(n+p) x[(n+p) x+k \beta]^{k-1} \\
& \times(n+p)(y-x)[(n+p)(y-x)+(j-k) \beta]^{j-k-1} e^{-[(n+p) y+j \beta]} \\
= & \sum_{k=0}^{\infty} \sum_{j=k}^{\infty} \omega\left(\frac{j}{n}\right) \frac{1}{k !(j-k) !}(n+p) x[(n+p) x+k \beta]^{k-1} \\
& \times(n+p)(y-x)[(n+p)(y-x)+(j-k) \beta]^{j-k-1} e^{-[(n+p) y+j \beta]} \\
= & \sum_{k=0}^{\infty} \sum_{l=0}^{\infty} \omega\left(\frac{k+l}{n}\right) \frac{1}{k ! l !}(n+p) x[(n+p) x+k \beta]^{k-1} \\
& \times(n+p)(y-x)[(n+p)(y-x)+l \beta]^{l-1} e^{-[(n+p) y+k \beta+l \beta]} .
\end{aligned}
$$

On the other hand, from (1.3), we have

$$
e^{(n+p)(y-x)}=\sum_{l=0}^{\infty} \frac{(n+p)(y-x)[(n+p)(y-x)+l \beta]^{l-1}}{l !} e^{-l \beta} .
$$

Therefore, $S_{n, p}^{\beta}(\omega ; x)$ may be written as

$$
\begin{aligned}
S_{n, p}^{\beta}(\omega ; x)= & \sum_{k=0}^{\infty} \omega\left(\frac{k}{n}\right) \frac{(n+p) x[(n+p) x+k \beta]^{k-1}}{k !} e^{-[(n+p) x+k \beta]} \\
= & \sum_{k=0}^{\infty} \omega\left(\frac{k}{n}\right) \frac{(n+p) x[(n+p) x+k \beta]^{k-1}}{k !} e^{-[(n+p) y+k \beta]} e^{(n+p)(y-x)} \\
= & \sum_{k=0}^{\infty} \sum_{l=0}^{\infty} \omega\left(\frac{k}{n}\right) \frac{1}{k ! l !}(n+p) x[(n+p) x+k \beta]^{k-1} \\
& \times(n+p)(y-x)[(n+p)(y-x)+l \beta]^{l-1} e^{-[(n+p) y+k \beta+l \beta]}
\end{aligned}
$$

Subtracting (4.4) from (4.3), we obtain

$$
\begin{aligned}
& S_{n, p}^{\beta}(\omega ; y)-S_{n, p}^{\beta}(\omega ; x) \\
= & \sum_{k=0}^{\infty} \sum_{l=0}^{\infty}\left\{\omega\left(\frac{k+l}{n}\right)-\omega\left(\frac{k}{n}\right)\right\} \frac{1}{k ! l !}(n+p) x[(n+p) x+k \beta]^{k-1} \\
& \times(n+p)(y-x)[(n+p)(y-x)+l \beta]^{l-1} e^{-[(n+p) y+(k+l) \beta]} .
\end{aligned}
$$


Using the semi-additivity property of $\omega$, we get

$$
\begin{aligned}
& S_{n, p}^{\beta}(\omega ; y)-S_{n, p}^{\beta}(\omega ; x) \\
\leq & \sum_{k=0}^{\infty} \frac{(n+p) x[(n+p) x+k \beta]^{k-1}}{k !} e^{-k \beta} \\
& \times \sum_{l=0}^{\infty} \omega\left(\frac{l}{n}\right) \frac{(n+p)(y-x)[(n+p)(y-x)+l \beta]^{l-1}}{l !} e^{-[(n+p) y+l \beta]} \\
= & e^{(n+p) x} \sum_{l=0}^{\infty} \omega\left(\frac{l}{n}\right) \frac{(n+p)(y-x)[(n+p)(y-x)+l \beta]^{l-1}}{l !} e^{-[(n+p) y+l \beta]} \\
= & \sum_{l=0}^{\infty} \omega\left(\frac{l}{n}\right) \frac{(n+p)(y-x)[(n+p)(y-x)+l \beta]^{l-1}}{l !} e^{-[(n+p)(y-x)+l \beta]} \\
= & S_{n, p}^{\beta}(\omega ; y-x),
\end{aligned}
$$

which shows the semi-additivity of $S_{n, p}^{\beta}$. From (4.5) it readily follows that $S_{n, p}^{\beta}(\omega ; y) \geq$ $S_{n, p}^{\beta}(\omega ; x)$ for $y \geq x$, i.e., $S_{n, p}^{\beta}$ is non-decreasing. Moreover, since the series is uniformly convergent, it follows that $\lim _{x \rightarrow 0^{+}} S_{n, p}^{\beta}(\omega ; x)=S_{n, p}^{\beta}(\omega ; 0)=\omega(0)=0$. This comples the proof.

\section{Monotonicity of the sequence of the Jain-Schurer operators}

In [5], Cheney and Sharma proved that the sequence of Szász-Mirakjan operators $P_{n}^{[0]}(f)$ is non-increasing in $n$, when $f$ is convex. The purpose of this section is to observe the monotonicity of the sequence of the Jain-Schurer operators when the attached function is convex and non-decreasing and $p \neq 0$. In the case $p=0$, we obtain monotonicity of the sequence of Jain operators in $n$ when $f$ is convex. For the proof, we further need the following Abel-Jensen formula

$$
(u+v+m \beta)^{m}=\sum_{k=0}^{m}\left(\begin{array}{c}
m \\
k
\end{array}\right)(u+k \beta)^{k} v[v+(m-k) \beta]^{m-k-1}
$$

for non-negative real number $\beta$, where $u, v \in \mathbb{R}$ and $m \geq 1$ (see, e.g., [21]). Reasoning as in [5], we present the following result:

Theorem 5.1. Let $f$ be a non-decreasing and convex function on $[0, \infty)$. Then, for all $n, S_{n, p}^{\beta}(f)$ is non-increasing in $n$ when $p \neq 0$. For the case $p=0$, the same result holds when $f$ is only convex on $[0, \infty)$.

Proof. From (1.3), with $\alpha=x$, it is obvious that

$$
e^{x}=\sum_{k=0}^{\infty} \frac{x(x+k \beta)^{k-1}}{k !} e^{-k \beta} .
$$


Since $S_{n, p}^{\beta}(f ; 0)=f(0)$, we study only for $x>0$. Taking the definition of $S_{n, p}^{\beta}$ and (5.2) into consideration, one has

$$
\begin{aligned}
& S_{n, p}^{\beta}(f ; x)-S_{n+1, p}^{\beta}(f ; x) \\
= & e^{x} \sum_{k=0}^{\infty} f\left(\frac{k}{n}\right) \frac{(n+p) x[(n+p) x+k \beta]^{k-1}}{k !} e^{-[(n+1+p) x+k \beta]} \\
& -\sum_{k=0}^{\infty} f\left(\frac{k}{n+1}\right) \frac{(n+1+p) x[(n+1+p) x+k \beta]^{k-1}}{k !} e^{-[(n+1+p) x+k \beta]} \\
= & \sum_{l=0}^{\infty} \frac{x(x+l \beta)^{l-1}}{l !} e^{-l \beta} \sum_{k=0}^{\infty} f\left(\frac{k}{n}\right) \frac{(n+p) x[(n+p) x+k \beta]^{k-1}}{k !} e^{-[(n+1+p) x+k \beta]} \\
& -\sum_{k=0}^{\infty} f\left(\frac{k}{n+1}\right) \frac{(n+1+p) x[(n+1+p) x+k \beta]^{k-1}}{k !} e^{-[(n+1+p) x+k \beta]} .
\end{aligned}
$$

By simple calculations, one can write

$$
\begin{aligned}
& \left(5.3 \oiint_{n, p}^{\beta}(f ; x)-S_{n+1, p}^{\beta}(f ; x)\right. \\
= & \sum_{l=0}^{\infty} \frac{x(x+l \beta)^{l-1}}{l !} e^{-l \beta} \sum_{k=l}^{\infty} f\left(\frac{k-l}{n}\right) \frac{(n+p) x[(n+p) x+(k-l) \beta]^{k-l-1}}{(k-l) !} e^{-[(n+1+p) x+(k-l) \beta]} \\
& -\sum_{k=0}^{\infty} f\left(\frac{k}{n+1}\right) \frac{(n+1+p) x[(n+1+p) x+k \beta]^{k-1}}{k !} e^{-[(n+1+p) x+k \beta]} \\
= & \sum_{k=0}^{\infty} e^{-[(n+1+p) x+k \beta]}\left\{\sum_{l=0}^{k} f\left(\frac{k-l}{n}\right) \frac{(n+p) x[(n+p) x+(k-l) \beta]^{k-l-1}}{(k-l) !} \frac{x(x+l \beta)^{l-1}}{l !}\right. \\
& \left.-f\left(\frac{k}{n+1}\right) \frac{(n+1+p) x[(n+1+p) x+k \beta]^{k-1}}{k !}\right\} \\
= & \sum_{k=0}^{\infty} e^{-[(n+1+p) x+k \beta]}\left\{\sum_{l=0}^{k} f\left(\frac{l}{n}\right) \frac{(n+p) x[(n+p) x+l \beta]^{l-1}}{l !} \frac{x[x+(k-l) \beta]^{k-l-1}}{(k-l) !}\right. \\
& \left.-f\left(\frac{k}{n+1}\right) \frac{(n+1+p) x[(n+1+p) x+k \beta]^{k-1}}{k !}\right\} \\
= & \sum_{k=0}^{\infty} \frac{(n+1+p) x[(n+1+p) x+k \beta]^{k-1}}{k !} e^{-[(n+1+p) x+k \beta]} \times \\
& \left\{\sum_{l=0}^{k}\left(\begin{array}{l}
k \\
l
\end{array}\right) \frac{(n+p) x[(n+p) x+l \beta]^{l-1} x[x+(k-l) \beta]^{k-l-1}}{(n+1+p) x[(n+1+p) x+k \beta]^{k-1}} f\left(\frac{l}{n}\right)-f\left(\frac{k}{n+1}\right)\right\}
\end{aligned}
$$

Now, it only remains to show that the curly bracket in the last formula must be non-negative. For this, we denote

$$
\alpha_{l}:=\left(\begin{array}{c}
k \\
l
\end{array}\right) \frac{(n+p) x[(n+p) x+l \beta]^{l-1} x[x+(k-l) \beta]^{k-l-1}}{(n+1+p) x[(n+1+p) x+k \beta]^{k-1}}>0,
$$

and

$$
x_{l}=\frac{l}{n}
$$


for $l=0,1, \ldots, k$. Now, replacing $u$ with $(n+p) x, v$ with $x, m$ with $k$ and $k$ with $l$ in (4.1) we evidently get

(5.4)

$\sum_{l=0}^{k} \alpha_{l}=\frac{1}{(n+1+p) x[(n+1+p) x+k \beta]^{k-1}}(n+1+p) x[(n+1+p) x+k \beta]^{k-1}=1$.

On the other hand, it follows that

$$
\begin{aligned}
\sum_{l=0}^{k} \alpha_{l} x_{l}= & \frac{1}{(n+1+p) x[(n+1+p) x+k \beta]^{k-1}} \times \\
& \sum_{l=0}^{k}\left(\begin{array}{c}
k \\
l
\end{array}\right) \frac{l}{n}(n+p) x[(n+p) x+l \beta]^{l-1} x[x+(k-l) \beta]^{k-l-1} \\
= & \frac{k(n+p) x}{n(n+1+p) x[(n+1+p) x+k \beta]^{k-1}} \times \\
& \sum_{l=0}^{k-1}\left(\begin{array}{c}
k-1 \\
l
\end{array}\right)[(n+p) x+\beta+l \beta]^{l} x[x+(k-l-1) \beta]^{k-l-2} .
\end{aligned}
$$

Making use of the Abel-Jensen formula given by (5.1) for $u=(n+p) x+\beta, v=$ $x, k=l, m=k-1,(5.5)$ reduces to

$$
\begin{aligned}
\sum_{l=0}^{k} \alpha_{l} x_{l} & =\frac{k(n+p) x}{n(n+1+p) x[(n+1+p) x+k \beta]^{k-1}}[(n+1+p) x+k \beta]^{k-1} \\
& =\frac{k(n+p)}{n(n+1+p)} .
\end{aligned}
$$

Taking into account (5.4), (5.6) and the convexity of $f,(5.3)$ reduces to

$$
\begin{aligned}
& \text { (5.7) } S_{n, p}^{\beta}(f ; x)-S_{n+1, p}^{\beta}(f ; x) \\
& =\sum_{k=0}^{\infty} \frac{(n+1+p) x[(n+1+p) x+k \beta]^{k-1}}{k !} e^{-[(n+1+p) x+k \beta]}\left\{\sum_{l=0}^{k} \alpha_{l} f\left(\frac{l}{n}\right)-f\left(\frac{k}{n+1}\right)\right\} \\
& \geq \sum_{k=0}^{\infty} \frac{(n+1+p) x[(n+1+p) x+k \beta]^{k-1}}{k !} e^{-[(n+1+p) x+k \beta]}\left\{f\left(\frac{k(n+p)}{n(n+1+p)}\right)-f\left(\frac{k}{n+1}\right)\right\} .
\end{aligned}
$$

It is obvious that when $p=0,(5.7)$ gives the non-negativity of $S_{n, 0}^{\beta}(f ; x)-$ $S_{n+1,0}^{\beta}(f ; x)$ under the convexity of $f$, which means that the sequence of Jain operators is non-increasing in $n$ under the convexity of the function. On the other hand, for $p \in \mathbb{N}$ it follows that

$$
\frac{k(n+p)}{n(n+1+p)}=\frac{k}{n+1} \frac{n+1}{n} \frac{n+p}{n+1+p}=\frac{k}{n+1} \frac{1+\frac{p}{n}}{\left(1+\frac{p}{n+1}\right)} .
$$


Hence, one has

$$
\frac{k(n+p)}{n(n+1+p)} \geq \frac{k}{n+1}
$$

by the fact that $\frac{1+\frac{p}{p}}{1+\frac{p}{n+1}} \geq 1$. Then, the result follows directly from the non-decreasingness of $f$.

\section{Acknowledgement}

The authors are extremely grateful to the referees for making valuable suggestions leading to the better presentation of the paper.

\section{R E F E R E N C E S}

1. U. ABEL and O. AGRATINI: Asymptotic behaviour of Jain operators. Numer. Algor. 71 (2016), 553-565.

2. O. AGRATINI: Approximation properties of a class of linear operators. Math. Meth. Appl. Sci. 36, (2013), no.17, 2353-2358.

3. F. ALTOMARE and M. CAMPITI: Korovkin-type approximaton theory and its applications. Walter de Gruyter, Berlin-New York, 1994.

4. M. BODUR, Ö. G. YILMAZ and A. ARAL: Approximation by Baskakov-SzászStancu Operators Preserving Exponential Functions. Constr. Math. Anal 1 (2018), no. $1,1-8$.

5. E. W. CHENEY and A. CHARMA: Bernstein power series. Can. J. Math. 16 (1964), 241-252.

6. N. ÇETIN and N. İSPIR: Approximation by complex modified Szász-Mirakjan operators. Studia Sci. Math. Hungar. 50 (2013), no. 3, 355-372.

7. E. DENIZ: Quantitative estimates for Jain-Kantorovich operators. Commun. Fac. Sci. Univ. Ank. Sér. A1 Math. Stat. 65 (2016), no. 2, 121-132.

8. R. A. DEVORE and G. G. LORENTZ: Constructive Approximation. Springer, Berlin, 1993.

9. A. FARCAŞ: An asymptotic formula for Jain's operators. Stud. Univ. BabeşBolyai Math. 57 (2012), no. 4, 511-517.

10. V. GUPTA and T. M. RASSIAS: Direct estimates for certain Szász type operators. Appl. Math. Comput. 251 (2015), 469-474.

11. V. GUPTA and G. C. GREUBEL: Moment estimations of new Szász-MirakyanDurrmeyer operators. Appl. Math. Comput. 271 (2015), 540-547.

12. G.C. JAIN: Approximation of functions by a new class of linear operators. J. Austral. Math. Soc. 13 (1972), no.3, 271-276.

13. Z. LI: Bernstein polynomials and modulus of continuity. J. Approx. Theory 102 (2000), no.1, 171-174.

14. A. J. LÓPEZ-MORENO, J. JÓDAR and F. J. MUÑOZ-DELGADO: Exponential type moments and localization results for multivariate Baskakov-Schurer operators. Int. J. Differ. Equ. Appl. 6 (2002), no.1, 53-67. 
15. H. N. MHASKAR and D. V. PAI: Fundamentals of approximation theory. CRC Press, Boca Raton, FL, Narosa Publishing House, New Delhi, 2000.

16. D. MICLĂUŞ and O. T. POP: The generalization of certain results for SzászMirakjan-Schurer operators. Creat. Math. Inform. 21 (2012), no. 1, 79-85.

17. V. N. MISHRA and P. SHARMA: On approximation properties of BaskakovSchurer-Szász operators. Appl. Math. Comput. 281 (2016), 381-393.

18. A. OLGUN, F. TAŞDELEN and A. ERENÇİ: A generalization of Jain's operators. Appl. Math. Comput. 266 (2015), 6-11.

19. F. SCHURER: On Linear positive operators in approximation theory. Thesis Delft, 1965.

20. P. C. SIKKEMA: On some linear positive operators. Indag. Math. 32 (1970), 327-337.

21. D. D. STANCU and E. I. STOICA: On the use Abel-Jensen type combinatorial formulas for construction and investigation of some algebraic polynomial operators of approximation, Stud. Univ. Babeş-Bolyai Math. 54 (2009), no.4, 167-182.

22. O. SZÁSZ: Generalization of S. Bernstein's polynomials to infinite interval. J. Research Nat. Bur. Standards 45 (1950), 239-245.

23. S. TARABIE: On Jain-Beta linear operators. Appl. Math. Inf. Sci. 6 (2012), no.2, 213-216.

Nursel Çetin

Turkish State Meteorological Service

Research Department

06560, Ankara, Turkey

nurselcetin07@gmail.com

Gülen Başcanbaz-Tunca

Ankara University

Faculty of Science

Department of Mathematics

06100, Tandogan Ankara, Turkey

tunca@science.ankara.edu.tr 